\title{
Nouvelles acquisitions en recherche molécu- laire dans les dysfonctions érectiles
}

\author{
Communication faite par K.E. ANDERSSON (Suède) au Congrès \\ Mondial sur l'Impuissance, Perth (Australie), Novembre 2000.
}

Compte rendu par E. AMAR

Il existe trois directions principales pour la recherche future dans la dysfonction érectile (D.E.) :

$1^{\circ}$ Les stratégies pour prévenir la dégénérescence caverneuse et/ou pour restaurer la fonction caverneuse.

$\left.2^{\circ}\right)$ La thérapie génique somatique.

$3^{\circ}$ ) Les nouvelles cibles moléculaires pour les traitements médicamenteux : la voie L-ARGININE - NO - cGMP (GMP cyclique) est la plus explorée, car la plus importante.

Il y a trois niveaux de cibles :

$1^{\circ} \mathrm{NO}$ - synthase

$2^{\circ}$ Guanylate - Cyclase

$3^{\circ}$ GMP Cyclique

avec trois différents récepteurs :

a) les protéines kinases,

b) les phosphodiestérases,

c) les canaux ioniques.

\section{LA NO SYNTHASE}

Il y a trois types d'enzymes :

- La NO Synthase neuronale (nNOS) la plus importante

- La NO Synthase endothéliale (eNOS)

- La NO Synthase inducible (iNOS)
Les NOS constitutionnelles (nNOS et eNOS) requièrent le substrat L-Arginine et des cofacteurs pour une activité optimale.

Les informations obtenues sur les résultats de l'action de L-Arginine chez les patients ayant une D.E. sont controversés pour l'instant.

Un cofacteur, le tétrahydrobiopterin (BH4) a démontré un effet positif améliorant la fonction endothéliale en cas d'hypercholestérolémie, de tabagisme chronique et de diabète. Son potentiel mérite d'être exploré.

\section{LA GUANYLATE CYCLASE}

La forme soluble de Guanylate Cyclase est la cible principale du NO (sGC). Elle consiste en quatre sous-unités et l'enzyme est activé jusqu'à 400 fois par le radical NO non chargé. Un nouvel instrument pour explorer la fonction de la sGC est le YC-1, une drogue qui se fixe sur le site allostérique de la molécule de sGC et la stimule indépendamment $d u$ NO. Cette drogue, de plus, semble sensibiliser la sGC à l'action du NO. Ceci peut ouvrir des nouvelles possibilités pour un développement médicamenteux.

Correspondance : E. Amar, 17 avenue Victor Hugo, 75116 PARIS 


\section{LE GMP CYCLIQUE}

Les signaux cGMP se font par trois différents récepteurs intra-cellulaires incluant les protéines kinases, les phosphodiestérases et les canaux ioniques.

\section{1) LES PROTÉINES KINASES CGMP RÉGULÉES :}

Deux différentes protéine-kinases cGMP dépendantes ont été identifiées chez les mammifères : cGK I et cGK II. L'inactivation de cGK1 chez la souris abolit à la fois à la relaxation du muscle lisse vasculaire et intestinal et l'inhibition de l'agrégation plaquettaire qui sont NO/cGMP dépendantes provoquant une hypertension, des troubles de la motricité intestinale et une hémostase anormale. Les souris cGK1 déficientes ont de très grandes difficultés à se reproduire.

Le tissu caverneux de ces souris a une incapacité ou une très réduite pour se relâcher en réponse au NO libéré par les neurones ou l'endothélium, ou administré de manière exogène.

L'analyse de la relaxation NO/cGMP induite montre clairement que la cGK1 est le médiateur principal de la cascade cGMP induite dans le tissu du corps caverneux. Son absence ne peut pas être compensée aussi efficacement par la cascade cAMP induite qui relâche le tissu érectile pénien normal et sans cGK1.

Tous ces éléments ensemble suggèrent que l'activation de la cGK1 est une étape clé dans la cascade des signaux conduisant à l'érection. Les anomalies de la fonction cGK1 pourraient entraîner une D.E. aussi chez les humains.

\section{2) LES PHOSPHODIESTÉRASES CGMP RÉGULÉES (PDE) :}

A cause de leur rôle capital dans la régulation $\mathrm{du}$ tonus musculaire lisse et les variations considérables des isoenzymes des Phosphodiestérases (13 PDE actuellement) en rapport avec les espèces et les tissus, les PDE sont devenues une cible attractive pour le développement des drogues. La PDE2, PDE3, PDE4, PDE5 et plusieurs autres isoenzymes ont été identifiés dans le tissu caverneux humain. Fonctionnellement PDE3A et PDE5A semblent les plus importantes. Récemment trois isoformes de la PDE5A (PDE5A 1-3) ont été identifiées.

Bien que la PDE5A 3 prédomine dans le tissu érectile, il reste à établir que des inhibiteurs sélectifs de la PDE5A3 apporteront un bénéfice par rapport au traitement par les inhibiteurs de la PDE5 existants !!!

\section{3) cGMP ET LES CANAUX POTAS- SIQUES :}

$\mathrm{Au}$ moins quatre canaux potassiques distincts ont été décrit dans le muscle lisse caverneux humain dont deux seulement sont intéressants :

- Un canal maxi-K Calcium sensible (Kca)

- Un canal K métaboliquement régulé (KATP) Les deux canaux Kca et KATP sont les mieux caractérisés et certainement les plus physiologiquement actifs.

Le canal maxi Kca apparaît être un point de convergence important pour moduler le degré de contraction du muscle lisse caverneux. L'activation de ce canal est très fortement augmentée à la suite de l'activation cellulaire aussi bien de la voie cAMP que cGMP. Il semble bien établi que ces seconds messagers (cAMP et cGMP) agissent pour moduler le tonus musculaire lisse caverneux (i.e relaxation) au moins en partie à travers l'activation de ce canal Kca. L'hyperpolarisation qui en résulte, à son tour, entraîne la diminution du flux transmembranaire du calcium à travers les canaux calciques L-Type Voltage dépendants et finalement la relaxation musculaire lisse.

La présence des protéines des canaux KATP a été retrouvé dans le corps caverneux humain et plusieurs études ont démontré que des activateurs potentiels du sous-type canal KATP entraînent une relaxation concentration dépendante du muscle lisse caverneux isolé.

Il est donc évident que l'exploration future du potentiel des ouvreurs sélectifs des canaux Kca et KATP du corps caverneux pourraient être fructueux. 


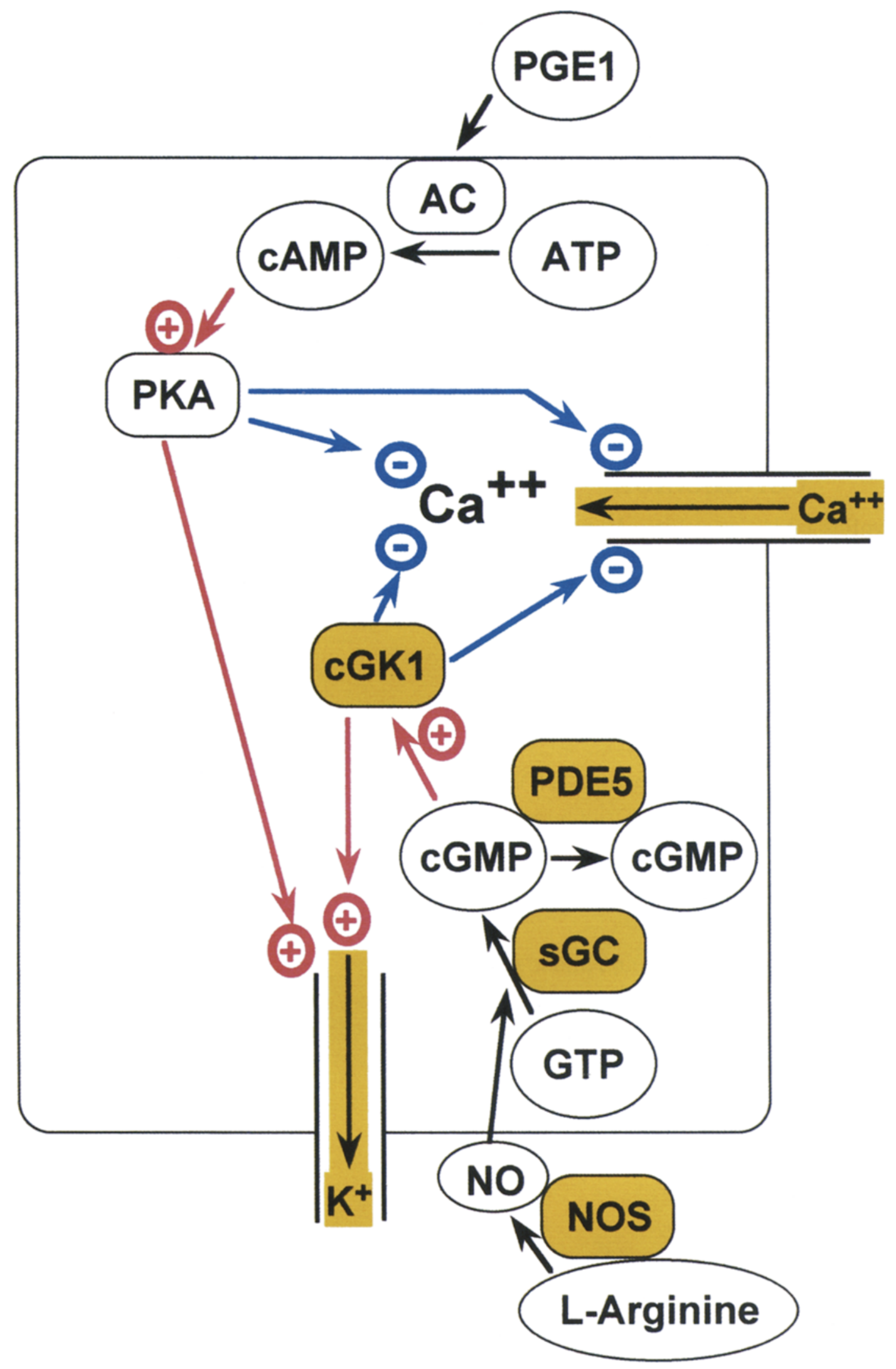




\section{4) CGMP ET CANAUX CALCIQUES :}

Plusieurs études ont démontré l'importance du flux calcique continu transmembranaire à travers les canaux calciques L-Type Voltagedépendant pour le maintien du tonus contractile du muscle lisse caverneux.

Il y a aussi de fortes preuves pour la présence et la signification physiologique du flux calcique transmembranaire à travers le canal calcique L-Type Voltage dépendant en réponse à l'activation avec l'endothéline 1 (récepteurs ET du sous type $A B$ ) et les agonistes des alpha adrénorécepteurs, événements très importants pour la fonction érectile.

\section{CONCLUSION}

De nouvelles cibles pour des drogues dans le traitement de la D.E., à l'intérieur ou influencé par la voie L-Arginine - NO - cGMP, peuvent être identifiés et demeurent un potentiel pour de futures investigations.

On pourrait résumer les cinq voies de recherche actuellement intéressantes (Figure 1) :

1) La régulation de la NO Synthase (NOS),

2) La stimulation de la Guanylate-Cyclase (sGC),

3) Linhibition spécifique des Phosphodiestérases,

4) La stimulation de la Protéine Kinase cGK1,

5) L'ouverture des Canaux Potassiques.

\section{Prospective in molecular research on erecti-} le dysfunction

\section{Summary of the communication of K.E. ANDERSSON (Sweden),}

$9^{\text {th }}$ World Meeting on impotence research, Perth (Australia), November 2000.

by E. AMAR 